\title{
A Vector Supersymmetry Killing the Infrared Singularity of Gauge Theories in Noncommutative space
}

Daniel N. Blaschke* and Manfred Schweda*

Institute for Theoretical Physics, Vienna University of Technology, Austria

E-mail: blaschke@hep.itp.tuwien.ac.at, mschweda@tph.tuwien.ac.at

\section{François Gieres}

Institut de Physique Nucléaire, Université Claude Bernard (Lyon 1), France

E-mail: gieres@ipnl.in2p3.fr

\section{Olivier Piguet ${ }^{\dagger} \ddagger$}

Univ. Federal do Espírito Santo, Brazil

E-mail: opiguet@yahoo.com

\begin{abstract}
We show that the "topological BF-type" term introduced by Slavnov in order to cure the infrared divergences of gauge theories in noncommutative space can be characterized as the consequence of a new symmetry. This symmetry is a supersymmetry, generated by vector charges, of the same type as the one encountered in Chern-Simons or BF topological theories.
\end{abstract}

Fifth International Conference on Mathematical Methods in Physics

24 - 28, April 2006

Rio de Janeiro, Brazil

\footnotetext{
*Work supported by "Fonds zur Förderung der Wissenschaftlichen Forschung” (FWF) under contract P15015-N08. †peaker.

¥Work supported in part by the Conselho Nacional de Desenvolvimento Científico e Tecnológico CNPq - Brazil.
} 


\section{Introduction}

The idea of noncommuting position and time coordinates [1] has been first introduced in the literature in Ref. [2]. In flat space-time, this amounts to postulate that Cartesian coordinate obey commutation relations

$$
\left[x^{\mu}, x^{v}\right]=i \theta^{\mu v}
$$

for some constant antisymmetric matrix $\theta^{\mu \nu}=-\theta^{v \mu}$. Classical fields $\varphi(x)$ thus become noncommuting because of the noncommutativity of $x$. An equivalent implementation of noncommutativity is through the Moyal [3] product, which is associative, but noncommutative:

$$
\varphi_{1}(x) * \varphi_{2}(x)=\left.e^{\frac{i}{2} \theta^{\mu v} \partial_{\mu}^{x} \partial_{\nu}^{y}}\left(\varphi_{1}(x) \varphi_{2}(y)\right)\right|_{y=x},
$$

where the coordinates are considered as commuting ${ }^{1}$. We consider a dimension $(1+3)$ space-time with Minkowski metric $\left(\eta_{\mu \nu}\right)=\operatorname{diag}(1,-1,-1,-1)$.

The field theory under consideration contains a U(1) gauge field $A_{\mu}$ and a scalar field $\lambda$ (Slavnov's field), with infinitesimal gauge transformations

$$
\delta A_{\mu}=\partial_{\mu} \varepsilon-i g\left[A_{\mu}, \varepsilon\right], \quad \delta \lambda=-i g[\lambda, \varepsilon]
$$

Notice the presence of commutators, $[X, Y]=X * Y-Y * X$, due to the noncommutativity of the Moyal product.

It is well known $[4,5,6,1]$ that such gauge theories in noncommutative space-time - which would be renormalizable in commutative space-time - suffer from infrared (IR) singularities mixed with the usual ultraviolet (UV) divergences.

Indeed, a gauge invariant action such as ${ }^{2}$

$$
S_{\text {Maxwell }}=-\frac{1}{4} \int d^{4} x F_{\mu v} F^{\mu v},
$$

possibly coupled with matter fields, with

$$
F_{\mu \nu}=\partial_{\mu} A_{v}-\partial_{v} A_{\mu}-i g\left[A_{\mu}, A_{v}\right],
$$

leads to infrared (IR) singularities associated with ultraviolet (UV) divergent Feynman diagrams. Typically, vacuum polarization graphs have IR singular parts

$$
\Pi_{\mathrm{IR}}^{\mu v}(k)=\frac{2 g^{2}}{\pi^{2}} \frac{\tilde{k}^{\mu} \tilde{k}^{v}}{\left(\tilde{k}^{2}\right)^{2}}, \quad \text { with } \tilde{k}^{\mu}=\theta^{\mu v} k_{v},
$$

and graphs with this insertion, such as the one shown in Fig. 1, are IR divergent.

Only special gauge theories are known to be free from these divergences (see e.g. the review [7]. Among them, let us mention Chern-Simons topological theory with particular couplings to matter [8], BF theories [9] and some supersymmetric Yang-Mills theories [10]. These theories are either topological [11], or supersymmetric [12]. A relevant question is to ask if nontopological gauge theories need to be supersymmetric in order to be free of IR singularities. A partial answer to this question is the object of the rest of this talk, which summarizes results presented in Ref. [13]

\footnotetext{
${ }^{1}$ From now on all field products will be Moyal ones, and the symbol $*$ will be ommitted.

${ }^{2}$ Remember that all products are Moyal.
} 


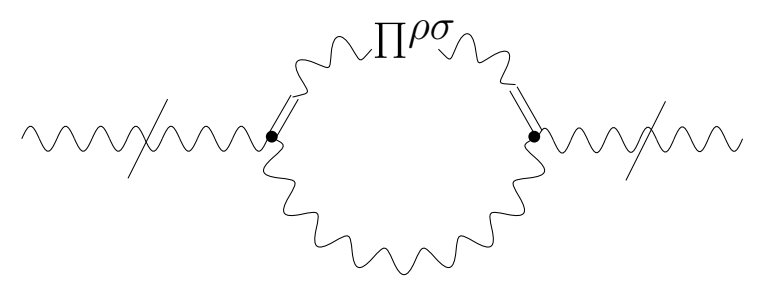

Figure 1: IR divergent graph with vacuum polarization insertion.

\section{Slavnov's modification of the noncommutative U(1) theory}

Slavnov $[14,15]$ has proposed a modification of the theory, adding to the action the term

$$
\frac{1}{2} \int d^{4} x \lambda \theta^{\mu v} F_{\mu v}
$$

which involves the scalar field $\lambda$ as a Lagrange multiplier. This term looks like a topological $B F$ action. It reduces the spin 1 gauge boson degrees of freedom to that of a spin 0 particle. Such a suppression of local degrees of freedom indeed happens in true topological theories, but there the suppression is complete. Slavnov has shown $[14,15,16]$ through a power-counting argument that IR singularities are absent in the theory obtained by adding the term (2.1) to the Maxwell action (1.4). We shall point out that the absence of IR singularity is in fact a consequence of the invariance of the theory under a vector supersymmetry.

In the following we shall choose the noncommutativity tensor to be space-like, in order to avoid problems with unitarity hence, without loss of generality, in the (1,2)-plane:

$$
\theta^{i j}=\theta \varepsilon^{i j}, \quad i, j=1,2 \quad\left(\theta^{12}=-\theta^{21}=1\right) .
$$

We use the notation $i, j, \cdots=1,2$ and $I, J, \cdots=0,3$.

The gauge invariant action thus reads

$$
S_{\mathrm{inv}}[A, \lambda]=\int d^{4} x\left(-\frac{1}{4} F_{\mu v}(x) F^{\mu v}(x)+\frac{1}{2} \lambda(x) \theta^{i j} F_{i j}(x)\right)
$$

with $F_{\mu \nu}$ given by (1.5).

\section{Gauge fixing and BRST symmetry}

Gauge fixing will conveniently be chosen axial, in the plane of the noncommutative coordinates. It is characterized by a vector $\left(n^{i}\right)=(0,1,0,0)$, a Lagrange multiplier field $B(x)$ and Faddeev-Popov ghosts $\bar{c}(x), c(x)$. The complete action is

$$
S=S_{\text {inv }}[A, \lambda]+S_{\mathrm{gf}}[A, B, c, \bar{c}],
$$

with

$$
S_{\mathrm{gf}}[A, B, c, \bar{c}]=\int d^{4} x\left(B(x) n^{i} A_{i}(x)-\bar{c}(x) n^{i} D_{i} c(x)\right)
$$

and

$$
D_{i}=\partial_{i} c-i g\left[A_{i}, c\right]
$$


This action is invariant under the BRST transformations

$$
\begin{array}{ll}
s A_{\mu}=D_{\mu} c, & s \bar{c}=B, \\
s \lambda=-i g[\lambda, c], & s B=0, \\
s c=\frac{i g}{2}[c, c], &
\end{array}
$$

the BRST operator $s$ being nilpotent: $s^{2}=0$.

\section{Vector supersymmetry, superalgebra and generalized BRST operator}

We note that the Slavnov term, together with the gauge-fixing terms, have the form of a 2dimensional gauge fixed topological $B F$ model, $\lambda$ playing the role of the " $B$ " field. Topological models of this kind ("Schwarz type" topological models) are known to possess a symmetry generated by a fermionic vector charge, called vector supersymmetry (VSUSY), responsible for their UV finiteness [17, 18, 19]. It turns out that this is true also here: the total gauge-fixed action is invariant under the VSUSY transformations

$$
\begin{array}{lll}
\delta_{i} A_{J}=0, & \delta_{i} A_{i}=0, & \delta_{i} \lambda=\frac{\varepsilon_{i j}}{\theta} n^{j} \bar{c}, \\
\delta_{i} c=A_{i}, & \delta_{i} \bar{c}=0, & \delta_{i} B=\partial_{i} \bar{c} .
\end{array}
$$

In the pure topological theories, the BRST and VSUSY generators form a closed algebra together with the translation generators. Here, in order to have a closed algebra, we must invoke an additional vector symmetry of the gauge-fixed action, peculiar to the present theory, with infinitesimal transformations

$$
\begin{aligned}
& \hat{d}_{i} A_{J}=-F_{i J}, \quad \hat{d}_{i} \lambda=-\frac{\varepsilon_{i j}}{\theta} \theta D_{K} F^{K j}, \quad(i=1,2) \\
& \hat{d}_{i} \Phi=0 \quad \text { for all other fields } .
\end{aligned}
$$

The algebra involving $s, \delta_{i}, \hat{d}_{i}$ and the (1-2)-plane translation generators $\partial_{i}$ is closed - modulo equations of motion:

$$
\left.\begin{array}{c}
{\left[\partial_{i}, s\right] \Phi=\left[\partial_{i}, \delta_{j}\right] \Phi=\left[\partial_{i}, \hat{d}_{j}\right] \Phi=0,} \\
{[s, s] \Phi=\left[s, \hat{d}_{j}\right] \Phi=0,} \\
{\left[\delta_{i}, \delta_{j}\right] \Phi=\left[\delta_{i}, \hat{d}_{j}\right] \Phi=0,}
\end{array}\right\} \quad \text { for all fields } \Phi,
$$




$$
\begin{aligned}
& {\left[\hat{d}_{i}, \hat{d}_{j}\right] A_{J}=\frac{\varepsilon_{i j}}{\theta} D_{J} \frac{\delta S}{\delta \lambda},} \\
& {\left[\hat{d}_{i}, \hat{d}_{j}\right] \lambda=\frac{\varepsilon_{i j}}{\theta} D_{J} \frac{\delta S}{\delta A_{J}},} \\
& {\left[\hat{d}_{i}, \hat{d}_{j}\right] \Phi=0 \quad \text { for } \Phi \in\left\{A_{i}, c, \bar{c}, B\right\} .}
\end{aligned}
$$

The various symmetries will be combined into a single generalized BRST operator $\Delta$, with the introduction of the constant ghosts $\xi^{i}, \varepsilon^{i}, \mu^{i}$ playing the role of the infinitesimal parameters of the symmetries $\partial_{i}, \delta_{i}, \hat{d}_{i}$. The statistics of these constant ghosts is fermionic, bosonic and fermionic, respectively. The generalized BRST operator thus reads

$$
\Delta=s+\xi^{i} \partial_{i}+\varepsilon^{i} \delta_{i}+\mu^{i} \hat{d}_{i},
$$

and its action on the various fields and on the constant ghosts is given by

$$
\begin{aligned}
& \Delta A_{i}=D_{i} c+\xi^{i} \partial_{i} A_{i}, \\
& \Delta A_{J}=D_{J} c+\xi^{i} \partial_{i} A_{J}+\mu^{i} F_{J i}, \\
& \Delta \lambda=-i g[\lambda, c]+\xi^{i} \partial_{i} \lambda+\varepsilon^{i} \frac{\varepsilon_{i j}}{\theta} n^{j} \bar{c}+\mu^{i} \frac{\varepsilon_{i j}}{\theta} D_{K} F^{j K}, \\
& \Delta c=\frac{i g}{2}[c, c]+\xi^{i} \partial_{i} c+\varepsilon^{i} A_{i}, \\
& \Delta \bar{c}=B+\xi^{i} \partial_{i} \bar{c}, \\
& \Delta B=\xi^{i} \partial_{i} B+\varepsilon^{i} \partial_{i} \bar{c}, \\
& \Delta \xi^{i}=D \mu^{i}=-\varepsilon^{i}, \quad \Delta \varepsilon^{i}=0 .
\end{aligned}
$$

$\Delta$ is nilpotent, but only on-shell:

$$
\begin{aligned}
& \Delta^{2} A_{i}=\varepsilon^{j} \frac{\varepsilon_{i j}}{\theta} \frac{\delta S}{\delta \lambda}, \\
& \Delta^{2} A_{J}=\frac{\mu^{i} \mu^{j}}{2} \frac{\varepsilon_{i j}}{\theta} D_{J} \frac{\delta S}{\delta \lambda}, \\
& \Delta^{2} \lambda=\frac{\mu^{i} \mu^{j}}{2} \frac{\varepsilon_{i j}}{\theta} D_{J} \frac{\delta S}{\delta A_{J}}+\varepsilon^{i} \frac{\varepsilon_{i j}}{\theta} \frac{\delta S}{\delta A_{j}}-\varepsilon^{i} \frac{1}{\theta^{2}} D_{i} \frac{\delta S}{\delta \lambda}, \\
& \Delta^{2} c=\Delta^{2} \bar{c}=\Delta^{2} B=0 .
\end{aligned}
$$

\section{Slavnov identity and ghost equations}

Useful Ward identities are consequences of the Slavnov-Taylor identity describing the invariance of the theory under the transformations (4.7). In order to write it, we associate an external field $\Phi^{*}$ - an "antifield" in the terminology of the authors of Ref. [20] - to the $\Delta$-variation of each of the fields $\Phi=A, \lambda, c$, respectively. The action $S_{\text {tot }}$ depending on the fields and antifields must be a solution of the Slavnov-Taylor identity [20,21]

$$
\begin{aligned}
\mathscr{S}\left(S_{\mathrm{tot}}\right) \equiv & \int d^{4} x\left(\sum_{\Phi \in\left\{A_{\mu}, \lambda, c\right\}} \frac{\delta S_{\mathrm{tot}}}{\delta \Phi^{*}} \frac{\delta S_{\mathrm{tot}}}{\delta \Phi}+\left(B+\xi^{i} \partial_{i} \bar{c}\right) \frac{\delta S_{\mathrm{tot}}}{\delta \bar{c}}+\left(\xi^{i} \partial_{i} B+\varepsilon^{i} \partial_{i} \bar{c}\right) \frac{\delta S_{\mathrm{tot}}}{\delta B}\right) \\
& -\varepsilon^{i}\left(\frac{\partial S_{\mathrm{tot}}}{\partial \xi^{i}}+\frac{\partial S_{\mathrm{tot}}}{\partial \mu^{i}}\right)=0 .
\end{aligned}
$$


The solution reads

$$
S_{\text {tot }}\left[A, \lambda, c, \bar{c}, B ; A^{*}, \lambda^{*}, c^{*} ; \xi, \mu, \varepsilon\right]=\int d^{4} x\left(B+\xi^{i} \partial_{i} \bar{c}\right) n^{i} A_{i}+\bar{S}\left[A, \lambda, c ; \hat{A}^{* i}, A^{* J}, \lambda^{*}, c^{*} ; \xi, \mu, \varepsilon\right],
$$

where $\hat{A}^{* i}=A^{* i}-n^{i} \bar{c}$, and

$$
\begin{aligned}
\bar{S}= & \int d^{4} x\left(-\frac{1}{4} F_{\mu v} F^{\mu v}+\frac{\theta}{2} \lambda \varepsilon^{i j} F_{i j}\right. \\
& +\hat{A}^{*^{i}}\left(D_{i} c+\xi^{i} \partial_{i} A_{i}\right)+A^{* J}\left(D_{J} c+\xi^{i} \partial_{i} A_{J}+\mu^{i} F_{J i}\right)+\lambda^{*}\left(-i g[\lambda, c]+\xi^{i} \partial_{i} \lambda+\mu^{i} \frac{\varepsilon_{i j}}{\theta} D_{K} F^{j K}\right) \\
& \left.+c^{*}\left(\frac{i g}{2}[c, c]+\xi^{i} \partial_{i} c+\varepsilon^{i} A_{i}\right)+\left(\frac{\mu^{i} \mu^{j}}{2} \frac{\varepsilon_{i j}}{\theta}\left(D_{J} A^{* J}\right)+\varepsilon^{i} \frac{\varepsilon_{i j}}{\theta} \hat{A}^{* j}-\varepsilon^{i} \frac{1}{2 \theta^{2}}\left(D_{i} \lambda^{*}\right)\right) \lambda^{*}\right) .
\end{aligned}
$$

Due to the axial gauge fixing, the field equations for $c$ and $\bar{c}$ take the form of local functional equations, namely, the antighost equation:

$$
\frac{\delta S_{\mathrm{tot}}}{\delta c}+i g\left[\bar{c}, \frac{\delta S_{\mathrm{tot}}}{\delta B}\right]=-n^{i} \partial_{i} \bar{c}+D_{\mu} A^{* \mu}-i g\left[\lambda, \lambda^{*}\right]+i g\left[c, c^{*}\right]+\xi^{i} \partial_{i} c^{*},
$$

and the ghost equation:

$$
\frac{\delta S_{\mathrm{tot}}}{\delta \bar{c}}+i g\left[c, \frac{\delta S_{\mathrm{tot}}}{\delta B}\right]-\xi^{i} \partial_{i} \frac{\delta S_{\mathrm{tot}}}{\delta B}=-n^{i} \partial_{i} c-\varepsilon^{i} \frac{\varepsilon_{i j}}{\theta} n^{j} \lambda^{*}
$$

Note that both right hand sides are linear in the dynamical fields. This fact expresses the well-kown freedom of the ghost fields in axial gauges [22].

\section{Ward identities of vector supersymmetry}

Interesting Ward identities may be extracted from the Slavnov-Taylor identity and from the ghost and antighost equations. E.g., a Ward identity for VSUSY is obtained by differentiating the Slavnov-Taylor identity (5.1) with respect to the VSUSY ghost $\varepsilon^{i}$. The result is

$$
\mathscr{W}_{i} S_{\text {tot }}=\Delta_{i}
$$

with

$$
\begin{aligned}
\mathscr{W}_{i} S_{\mathrm{tot}}=\int d^{4} x( & \partial_{i} \bar{c} \frac{\delta S_{\mathrm{tot}}}{\delta B}+A_{i} \frac{\delta S_{\mathrm{tot}}}{\delta c}+\left(\frac{\varepsilon_{i j}}{\theta}\left(n^{j} \bar{c}-A^{* j}\right)+\frac{1}{\theta^{2}} D_{i} \lambda^{*}\right) \frac{\delta S_{\mathrm{tot}}}{\delta \lambda} \\
& \left.+\lambda^{*} \frac{\varepsilon_{i j}}{\theta} \frac{\delta S_{\mathrm{tot}}}{\delta A_{j}}+\left(c^{*}+\frac{i g}{\theta^{2}} \lambda^{*} \lambda^{*}\right) \frac{\delta S_{\mathrm{tot}}}{\delta A^{* i}}\right),
\end{aligned}
$$

and

$$
\Delta_{i}=\frac{\partial S_{\mathrm{tot}}}{\partial \xi^{i}}+\frac{\partial S_{\mathrm{tot}}}{\partial \mu^{i}}+\int d^{4} x \frac{\varepsilon_{i j}}{\theta} n^{j}\left(B+\xi^{i} \partial_{i} \bar{c}\right) \lambda^{*}
$$

We note that the breaking term $\Delta_{i}$ vanishes at vanishing antifields. 
Knowing that the total action $S_{T}\left[\Phi, \Phi^{*}, \cdots\right]$ is the functional generator of the vertex functions (1-particle irreducible amputated graph contributions) in the tree graph approximation, and that the Legendre transform

$$
Z^{\mathrm{c}}\left[J_{\Phi}, \Phi^{*}, \cdots\right]=S_{\mathrm{tot}}\left[\Phi, \Phi^{*}, \cdots\right]+\sum_{\Phi} \int J_{\Phi} \Phi, \quad \text { with } \quad J_{\Phi}=-\frac{\delta S_{\mathrm{tot}}}{\delta \Phi},
$$

yields the functional generator of the connected Green functions, we obtain Ward identities for the connected Green functions - here in the tree approximation. The Ward identity for VSUSY at vanishing antifields, which reads, for the vertex functions, as

$$
\int d^{4} x\left(\partial_{i} \bar{c} \frac{\delta S_{\mathrm{tot}}}{\delta B}+A_{i} \frac{\delta S_{\mathrm{tot}}}{\delta c}+\frac{\varepsilon_{i j}}{\theta} n^{j} \bar{c} \frac{\delta S_{\mathrm{tot}}}{\delta \lambda}\right)=0
$$

yields, for the connected Green functions,

$$
\int d^{4} x\left(J_{B} \partial_{i} \frac{\delta Z^{c}}{\delta J_{\bar{c}}}-J_{c} \frac{\delta Z^{c}}{\delta J_{A}^{i}}+\frac{\varepsilon_{i j}}{\theta} n^{j} J_{\lambda} \frac{\delta Z^{c}}{\delta J_{\bar{c}}}\right)=0 .
$$

Differentiating the latter, e.g. with respect to $J_{c}$ and to $J_{A}^{v}$, yields, for the gauge field propagator, the condition

$$
\Delta_{A_{i} A_{\mu}}=0 .
$$

Other consequences of VSUSY are obtained from the Ward identity for vertex functions, differenciated with respect to $A_{\mu}$ and $A_{J}$, or $A_{i}$ and $A_{j}$ :

$$
\Gamma_{\lambda A_{\mu} A_{J}}(x, y, z)=0
$$

and

$$
\Gamma_{\lambda A_{i} A_{j}}(x, y, z)=i g \theta \varepsilon^{k l} K(x, y, z),
$$

where

$$
K(x, y, z)=\left.e^{\frac{i}{2} \theta \varepsilon^{i j} \partial_{i}^{x} \partial_{j}^{u}}(\delta(x-y)-\delta(u-z)-\delta(x-z)-\delta(u-y))\right|_{u=x} .
$$

If we assume the latter result, which coincides with the tree vertex deduced from the classical action $S_{\text {tot }}$, to be valid for the quantized theory, we would conclude that the vertex $\Gamma_{\lambda A_{i} A_{j}}$ would not adquire radiative corrections.

\section{Cancellation of the IR singularities}

Let us now show through a graphical analysis how the IR singularities are cancelled as a consequence of the VSUSY Ward identities. We first see, from Fig. 2, that the $\lambda A A$-vertex contracted with a $A A$-propagator vanishes because of (6.2). Secondly, looking at Fig. 3, we observe that one cannot build a Feynman loop graph containing a $\lambda A A$-vertex without the presence of at least one $A A$-propagator. It then follows that loop corrections to the $\lambda \lambda$ and $\lambda A$ propagators vanish.

More generally, we can conclude that all loop graphs involving a $\lambda A A$ vertex vanish. In particular, dangerous vacuum polarization insertions as in Fig. 1 cancel. Finally, contributions of IR singular parts of vertices $\Gamma_{A_{\mu_{1}} \cdots A_{\mu_{N}}}(N \geq 2)$ connected to $A A$-propagators in loop graphs vanish, 


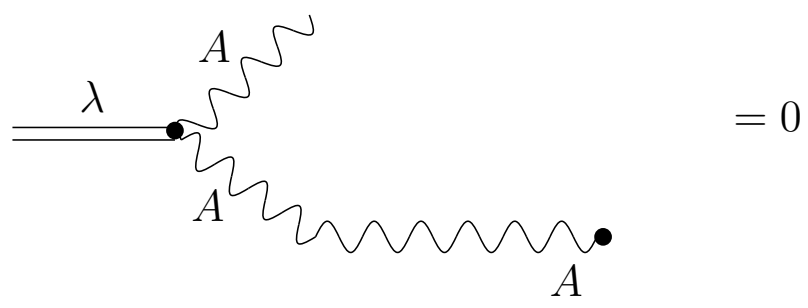

Figure 2: The $\lambda A A$-vertex contracted with a photon propagator.

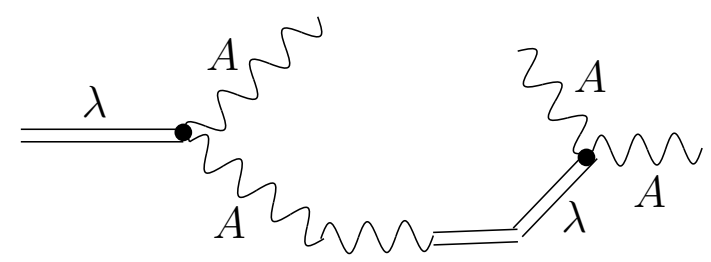

Figure 3: Trying to build loop graphs with a $\lambda A A$-vertex and $A \lambda$-propagators, but without $A A$-propagators.

too, since these singularities are present in vertices with indices $i=1,2$ only. E.g. in the singular part (1.6) of the vacuum polarization, the indices $\mu$ and $v$ take the values 1 or 2 due to our choice of the noncommutativity matrix $\theta$.

In conclusion, no IR singularities are left.

\section{Conclusions and outlooks}

We can conclude from our analysis that Poincaré supersymmetry is not needed in order to cure the IR-UV mixing in gauge theories constructed in noncommutative space.

However the concept of supersymmetry, manifest in the form of VSUSY, seems to play a decisive role in theories which are not Poincaré supersymmetric. Indeed, as we have seen in our case, the Ward identities of VSUSY yield exactly the propagator and vertex properties which are needed for cancelling the IR singularities.

What is the role of VSUSY with respect to the IR-UV mixing in topological gauge theories in general remains an open question.

Finally, the study of more general theories, based on a rigorous quantization scheme (perturbative [21] or not) seems desirable.

\section{References}

[1] M.R. Douglas and N.A. Nekrasov, Noncommutative field theory, Rev. Mod. Phys. 73 (2001) 977;

R.J. Szabo, Quantum field theory on noncommutative spaces, Phys. Rep. 378 (2003) 207.

[2] H.P. Snyder, Quantized space-time, Phys. Rev. 71 (1947) 38.

[3] H.J. Groenewold, On the principles of elementary quantum mechanics, Physica (Utrecht) 12 (1946) 405;

J.E. Moyal, Quantum mechanics as a statistical theory, Proc. Cambridge Phil. Soc. 45194999. 
[4] T. Filk, Divergences in a field theory on quantum space, Phys. Lett. B376 (1996) 53.

[5] A. Matusis, L. Susskind and N. Toumbas, The IR/UV connection in the non-commutative gauge theories, JHEP 12 (2000) 002, [hep-th/0002075].

[6] M. Attems, D. N. Blaschke, M. Ortner, M. Schweda, S. Stricker and M. Weiretmayr, Gauge independence of IR singularities in non-commutative QFT - and interpolating gauges, JHEP 07 (2005) 071, [hep-th/0506117];

M. Hayakawa, Perturbative analysis on infrared and ultraviolet aspects of noncommutative QED on $\mathbf{R}^{4}$, [hep-th/9912167];

F. R. Ruiz, Gauge-fixing independence of IR divergences in non-commutative $U(1)$, perturbative tachyonic instabilities and supersymmetry, Phys. Lett. B502 (2001) 274, [hep-th/ 012171 ] .

[7] M. Gomes, Remarks on noncommutative field theories, Braz. J. Phys. 34 (2004) 1330.

[8] A. A. Bichl, J. M. Grimstrup, V. Putz and M. Schweda, Perturbative Chern-Simons theory on noncommutative $\mathbf{R}^{3}$, JHEP 07 (2000) 046, [ hep-th/ 0004071 ] .

[9] A. Blasi, N. Maggiore and M. Montobbio, Instabilities of noncommutative two-dimensional BF model, Mod. Phys. Lett. A20 (2005) 2119, [hep-th/ 0504218 ];

A. Blasi, N. Maggiore and M. Montobbio, Noncommutative two-dimensional BF model, [hep-th/0512006].

[10] E. A. Asano, L. C. T. Brito, M. Gomes, A. Y. Petrov and A. J. da Silva, Consistent interactions of the 2+1 dimensional noncommutative Chern-Simons field, Phys. Rev. D71 (2005) 105005, [hep-th/0410257].

[11] D. Birmingham, M. Blau, M. Rakowski and G. Thompson, Topological Field Theory, Phys. Rep. 209 (1991) 129.

[12] J. Wess and J. Bagger, Supersymmetry and Supergravity, 2nd Rev., Princeton Series in Physics, Princeton Univ Press (1992).

[13] D. N. Blaschke, F. Gieres, O. Piguet and M. Schweda, A vector supersymmetry in non-commutative U(1) gauge theory with the Slavnov term, J. High Energy Phys. 0605 (2006) 059, [hep-th/0604154].

[14] A. A. Slavnov, Consistent noncommutative quantum gauge theories?, Phys. Lett. $\mathbf{B 5 6 5}$ (2003) 246, [hep-th/0304141].

[15] A. A. Slavnov, Gauge-invariant U(1) model in the axial gauge on the noncommutative plane, Teor. Mat. Fiz. 140N3 (2004) 388.

[16] D. N. Blaschke, S. Hohenegger and M. Schweda, Divergences in non-commutative gauge theories with the Slavnov term, JHEP 11 (2005) 041, [hep-th/ 0510100 ].

[17] F. Delduc, F. Gieres and S. P. Sorella, Supersymmetry of the $d=3$ Chern-Simons action in the Landau gauge, Phys. Lett. B225 (1989) 367;

F. Delduc, C. Lucchesi, O. Piguet and S. P. Sorella, Exact scale invariance of the Chern-Simons theory in the Landau gauge, Nucl. Phys. $B 346$ (1990) 313;

E. Guadagnini, N. Maggiore and S. P. Sorella, Supersymmetric structure of four-dimensional antisymmetric tensor fields, Phys. Lett. B255 (1991) 65.

[18] A. Blasi, O. Piguet and S. P. Sorella, Landau gauge and finiteness, Nucl. Phys. B356 (1991) 154. 
[19] S. Emery, M. Kruger, J. Rant, M. Schweda and T. Sommer, Two-dimensional BF model quantized in the axial gauge, Nuovo Cim. A111 (1998) 1321, [hep-th/9609240] ;

O. M. Del Cima, K. Landsteiner and M. Schweda, Twisted $N=4$ SUSY algebra in topological models of Schwarz type, Phys. Lett. B439 (1998) 289, [hep-th/9806137 ] ;

O. M. Del Cima, J. M. Grimstrup and M. Schweda, On the finiteness of a new topological model in D = 3, Phys. Lett. B463 (1999) 48, [hep-th/9906146];

F. Gieres, J. Grimstrup, T. Pisar and M. Schweda, Vector supersymmetry in topological field theories, JHEP 06 (2000) 018, [hep-th/ 0002167 ] .

[20] I. A. Batalin and G. A. Vilkovisky, Gauge algebra and quantization, Phys. Lett. B102 (1981) 27; I. A. Batalin and G. A. Vilkovisky, Quantization of gauge theories with linearly dependent generators, Phys. Rev. D28 (1983) 2567.

[21] O. Piguet and S. P. Sorella, Algebraic Renormalization: Perturbative Renormalization, Symmetries and Anomalies, Lect. Notes Phys. M28 (Springer, 1995);

A. Boresch, S. Emery, O. Moritsch, M. Schweda, T. Sommer and H. Zerrouki, Applications of Noncovariant Gauges in the Algebraic Renormalization Procedure (World Scientific, 1998).

[22] W. Kummer, Eichtransformation und Quantelung des freien elektromagnetischen Feldes, Acta Phys. Austriaca 14 (1961) 149;

W. Kummer, Ghost free nonabelian gauge theory, Acta Phys. Austriaca 41 (1975) 315. 